\title{
Correction to: Use of the Er,Cr:YSGG laser for removing remnant adhesive from the enamel surface in rebonding of orthodontic brackets
}

\author{
Katsunori Koide ${ }^{1} \cdot$ Satoshi Tanaka ${ }^{2}$ Toshiya Endo ${ }^{1,3}$
}

Published online: 5 March 2020

(c) The Society of The Nippon Dental University 2020

\section{Correction to: Odontology \\ https://doi.org/10.1007/s10266-019-00448-0}

In the original publication of the article, Fig. 2 was published incorrectly. The correct version of Fig. 2 is provided below.
Publisher's Note Springer Nature remains neutral with regard to jurisdictional claims in published maps and institutional affiliations.

The original article can be found online at https://doi.org/10.1007/ s10266-019-00448-0.

Katsunori Koide

albert@ngt.ndu.ac.jp

1 Orthodontics and Dentofacial Orthopedics, Field of Oral and Maxillofacial Growth and Development, Course of Clinical Science, The Nippon Dental University Graduate School of Life Dentistry at Niigata, 1-8 Hamaura-cho, Chuo-ku, Niigata 951-8580, Japan

2 Department of Pediatric Dentistry, The Nippon Dental University School of Life Dentistry at Niigata, 1-8 Hamaura-cho, Chuo-ku, Niigata 951-8580, Japan

3 Department of Orthodontics, The Nippon Dental University School of Life Dentistry at Niigata, 1-8 Hamaura-cho, Chuo-ku, Niigata 951-8580, Japan 
Fig. 2 Enamel surface roughness measurements for the Er,Cr:YSGG laser removal system (square data points) and the plier removal system (diamond data points).

Vertical bars represent standard deviations for enamel surface roughness measurements of the Er,Cr:YSGG laser removal system (solid lines) and the plier removal system (dotted lines). $* * * P<0.001$

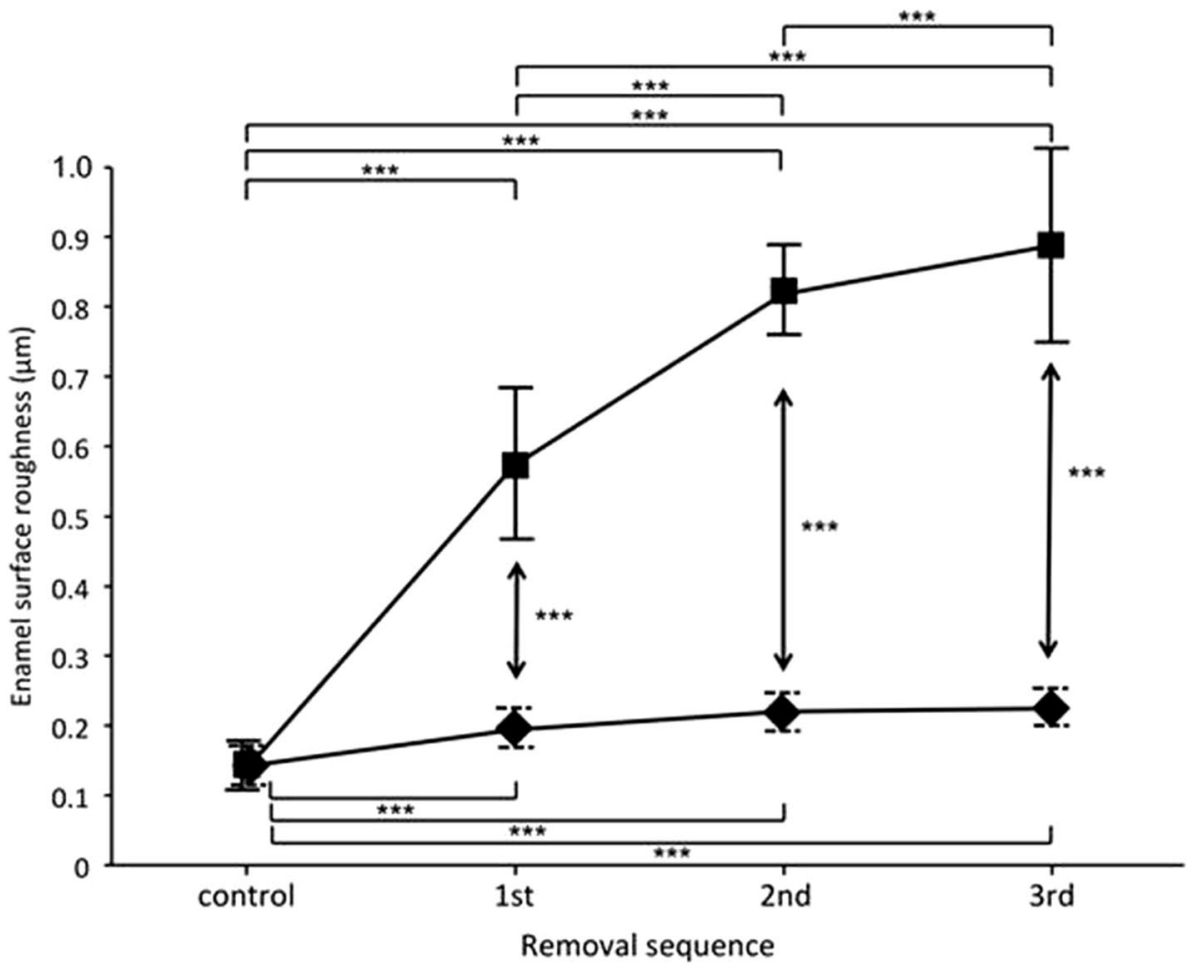

